\title{
Synthesis and Biological Interest of Structured Docosahexaenoic Acid-Containing Triacylglycerols and Phospholipids
}

\author{
Madeleine Picq*, Nathalie Bernoud-Hubac and Michel Lagarde
}

Université de Lyon, Inserm UMR 1060 (CarMeN), Univ-Lyon 1, Inra, INSA-Lyon, IMBL, Villeurbanne, France

\begin{abstract}
In light of rapid progress in biochemistry and modern bioengineering, there is a great interest in understanding how modifying the structure of naturally occurring lipids can be used to improve their nutritional and health properties. Structured lipids (SLs) or custom-made lipids can supply functional fatty acids because of their specific positioning in the glycerol structure. Health benefits of n-3 fatty acids such as docosahexaenoic acid (DHA) have been widely reported. Little information is available on the potential for health benefits of the SLs molecules that are rich in DHA and have well defined structure.This review attempts to summarize our present state of knowledge of various approaches to produce structured DHA-containing triglycerides and phospholipids as well as their applications.
\end{abstract}

Keywords: Chemistry, DHA, Lipids, Fat, Lipase, Nutrition.

\section{INTRODUCTION}

Lifestyle-related diseases such as atherosclerosis, hyperlipidemia, diabetes and hypertension, and prevalent diseases in industrial countries such as cancer and neurodegenerative diseases are increasing. Attributed to over consumption of fats, obesity has especially emerged as one of the major cardiovascular risk factor according to epidemiologic studies [1,2]. The management of the vascular risk factor together with the life-style changes have been reported to delay the onset of the dementia syndromes such as Alzheimer's disease [3]. As a result, there is an increased awareness by consumers of their needs to reduce the intake of calories derived from fats and modify their dietary habits. As saturated fat intake is associated with increased risk for obesity, high blood cholesterol, coronary heart disease, cancer and arteriosclerosis, the quality of dietary lipids could decrease the mortality of the life-style diseases [4]. A great number of in vitro and in vivo investigations have shown pharmacological and nutritional effects for long-chain n-3 polyunsaturated fatty acids (LC n-3 PUFAs) from fish oils, such as docosahexaenoic (DHA; 22:6n-3) and eicosapentaenoic (EPA; 20:5n-3) acids [5]. They exert beneficial effects on chronic inflammatory diseases [6] and possess hypolipidemic effects [7,8]. They have been shown attractive in the treatment of patients with diabetes [9], and nutritional DHA supplementation is also effective in preventing experimental diabetic neuropathy [10]. In a recent review by Mozaffarian [11], the conclusion was that n-3 PUFAs reduce the risk of cardiac death. Recent studies suggested that n-3 PUFAs could be cancer preventive and suppressive agents, and then used as auxiliary agents for cancer therapy [12]. It is now recognized that DHA is important for the acquisition of ocular vision and brain development in infants. So, there are strong demands for DHA concentrates by the pharmaceutical as well as health food industries, for use as food supplements. Humans have limited ability to convert the n-3 PUFA precursor linolenic acid, contained in vegetable oils, into DHA. Currently, seafood is the primary dietary source of this PUFA. However, global fish stocks are declining and

*Address correspondence to this author at the Inserm UMR 1060 (CarMeN), 20 Ave A. Einstein, Bldg Pasteur-IMBL, 69621 Villeurbanne, France; Tel: 33(0)472438574; Fax: 33(0)472438524; E-mail: madeleine.picq@insa-lyon.fr are not expected to meet future nutritional needs. A logical strategy is to develop lipid products, especially those with specific structure and high purity to meet the particular nutritional and pharmaceuti- cal requirements [13-15]. The concept of structured lipids (SLs) for nutritional and medical use was first introduced in 1987 by Babayan [16]. The term SLs refers to lipids which are re-structured, in order to chemically or enzymatically modify the composition and /or position of their fatty acids (FAs), in contrast to natural oils and fats. The latter are simply mixtures of a number of molecular species of triglycerides (TAGs) or phospholipids (PLs), different in terms of FA species (saturated, monounsaturated or polyunsaturated) and position distribution on the glycerol backbone, and types of polar head in the case of PLs.

In this review, we provide a concise and up-to-date survey of novel enzymatic and chemical approaches to modify TAGs and PLs in order to obtain DHA-enriched TAGs and PLs, as well as an overview on the interest of these SLs.

\section{SYNTHESIS OF STRUCTURED TRIACYLGLYCEROLS (STAGS)}

\subsection{Synthesis of STAGs from Natural Oils and Fats}

TAG results from esterification of glycerol by three FAs. Each TAG may contain different fatty acyls or partially identical ones. TAGs are naturally synthesised by enzymes (acyl transferases) which have specificity for positions sn-1 and sn-2 of L-glycerolphosphate, and for the sn-3 position following the phosphate removal. Therefore in plants and animals, specific fatty acyls occupy specific positions within the TAG molecule depending on the species. SLs that have a pre-determined composition and distribution of FAs on the glycerol backbone have recently gained considerable attention as nutritional and health supplements. Since there is emerging evidence of beneficial PUFAs, it is important to substitute saturated FAs (SFAs) with PUFAs, but a balance in the dietary fat containing PUFAs, SFAs and monounsaturated FAs (MUFAs) present in significant quantities in many sources must be stated. World Health Organization recommends Oil Technologists and Nutritionists an ideal oil which would have the S/M/P ratio of 1/1/1 [17]. Like the degree of unsaturation, the geometry of double bonds (E or Z) and FA chain length are important. Medium-chain FAs 


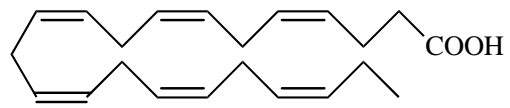

Fig. (1). Structure of docosahexaenoic acid (DHA).

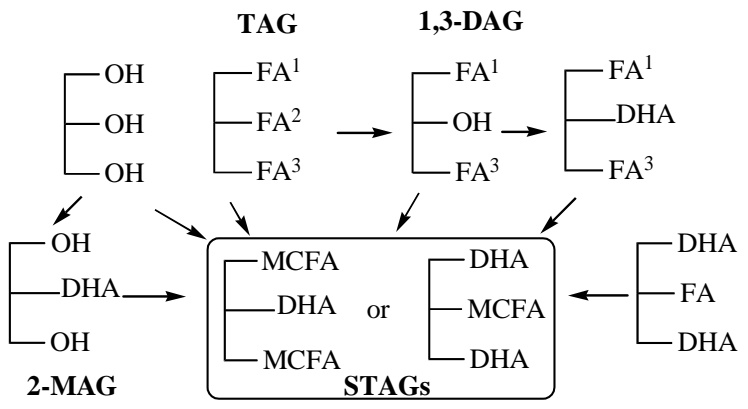

Fig. (2). Scheme for producing structured triacylglycerols (STAGs). Where $\mathrm{FA}=$ fatty acid, MCFA = medium-chain fatty acid, MAG = monoacylglycerol, DAG $=$ diacylglycerol.

(MCFAs) with C6-C12 carbon chain lengths are more rapidly metabolized than long-chain FAs (LCFAs) with C14-C24 carbon chain lengths, thus providing a rapid source of energy. This has led to the development of a new type of SLs in food industries called medium and long-chain TAGs (MLCTs). Usually, STAGs have LC-PUFAs located at the internal position with MCFAs at the external positions. These SLs are made by modifying natural oils or fats and, depending on the type of substrates available, chemical or enzymatic reactions can be used to synthesize them. In the process of random chemical inter-esterification, the fat is heated (at about 100-

$140^{\circ} \mathrm{C}$ ) for a short period of time, in the presence of a catalyst (e.g. sodium methoxide). The FAs are released from the glycerol backbone and re-esterified in a random manner within the three sn positions. Chemical inter-esterification is simple, inexpensive, and easy to scale-up, but is not capable of modifying specific positions due to the random nature of reactions [18]. In addition, it may produce side products that are hard to eliminate and is likely to alter the all- cis framework of PUFAs. As a matter of fact, EPA and DHA may be drastically altered by oxidation, Z-E isomerisation, double-bond migrations or polymerization [19].

In contrast, the reactions catalyzed by enzymes such as lipases or phospholipases are more promising for position-specific modifications of lipids. They offer high efficiency and mildness and their applications in organic media is established.

Lipase-catalyzed enzymatic approaches are: inter-esterification, acidolysis and esterification reactions. Inter-esterification consists in exchange of fatty acyl moieties within (intra-esterification) and among (inter-esterification) TAGs molecules. Enzyme-directed interesterifications are used to create TAGs with specific positional composition using enzymes with regio-specificity from vegetable oil like coconut, palm kernel, soybean or rapeseed oil. Acidolysis or inter-esterification methods are acyl exchange in oils with excess of FA or its ethylester respectively.

Acidolysis: $\mathrm{R}_{1} \mathrm{COOR}+\mathrm{R}_{2} \mathrm{COOH} \longrightarrow \mathrm{R}_{2} \mathrm{COOR}+\mathrm{R}_{1} \mathrm{COOH}$

Inter-esterification: $\mathrm{R}_{1} \mathrm{COOR}_{2}+\mathrm{R}_{3} \mathrm{COOR}_{4} \longrightarrow \mathrm{R}_{1} \mathrm{COOR}_{4}+$ $\mathrm{R}_{3} \mathrm{COOR}_{2}$

Choosing the right type of enzyme is important, and 1,3specific immobilized enzymes may be used to be sure that the essential FA is maintained at the sn- 2 position, creating the desired structure of MLCTs. These reactions applied to cod liver oil, with high EPA and/or DHA concentration, in presence of 1,3-regio- specific immobilized lipase from the fungus Mucor miehei resulted in TAGs containing 60-65\% EPA or DHA. Despite the 1,3-regiospecificity of the lipase, the internal position became enriched to equal extent as the external positions due to non-enzymatic acylmigration processes [20]. Soybean oils can be substrates for producing DHA-enriched TAGs using two immobilized lipases from Rhizomисо miehei and Candida antartica [21]. There is an extensive literature concerning incorporation of MCFAs in PUFA-rich fish oil via lipase-assisted acidolysis [22-26].

Hamam and Shaihidi [27] produced STAGs via acidolysis of DHA single cell oil derived from Crypthecodinium cohnii microalgae with capric acid (C10:0 CA) using five commercially available lipases. Among these lipases PS-30 from Pseudomonas $s p$ was the most effective to produce TAGs with CA present mainly at the sn-1 and sn-3 positions, whereas DHA was present at the sn- 2 position, although partially located at the sn- 1 and sn-3 positions. Xu and al [28] used an ultra-filtration membrane reactor for acidolysis between fish oil concentrates and medium-chain TAGs (MCTs) with immobilized Rhizomucor miehei lipase (lipozyme IM) as biocatalyst. The membrane was used to separate released MCFAs from the mixture during the reaction. Thus, incorporation of EPA/DHA into MCTs was increased by about $15 \%$. Garcia et al. [29] have tested five lipases in a reaction medium composed solely of substrates and either free or immobilized commercial lipase preparation, and successfully incorporated conjugated linoleic acid (CLA) in Menhaden oil, a rich source of $n-3$ FAs.

Acidolysis is the simplest and most direct route for the synthe- sis of MLCTs. Nevertheless, acyl-migration is a major problem in batch reactors causing a decrease in the yield of the target TAGs. To prevent it, some authors relate one alternative process in two steps. First step was production of 2-monoacyl-glycerol (2-MAG) by enzymatic alcoholysis of fish oils and second step was enzymatic esterification of the external positions of the 2-MAG using sn-1,3 specific lipases.

\section{Alcoholysis: $\mathrm{R}_{1} \mathrm{COOR}+\mathrm{R}_{2} \mathrm{OH} \longrightarrow \mathrm{ROH}+\mathrm{R}_{1} \mathrm{COOR}_{2}$}

Some studies using oils with a high DHA content show that fungal sn-1,3 lipases scarcely act on DHA residues at the sn-1- and 3-positions especially when immobilized Rhizomисor miehei was used. Irimescu et al. [30] reported that lipase from Candida antartica (Novozym 435) presents high 1,3-regio-specific activity toward tri-docosahexaenoyl-glycerol in the ethanolysis step to produce 2DHA-MAG with a high excess of ethanol. So, spontaneous acyl migration was minimized since 2-DHA-MAG needs not to be purified before its use in the re-esterification step. Then, using the same conditions for ethanolysis of bonito oil, the authors obtained 2DHA-MAG which was re-esterified with ethyl caprylate (CA) by lipozyme IM [31]. By this way they produced $85 \%$ TAGs rich in DHA with CA at the external positions, but also side-products like TAGs with only one CA residue or tri-capryloyl-glycerol. More recently, another team studied the factors that influence the ethanolysis of cod liver and tuna oils, and esterification of 2-MAGs with CA. The authors reported that during the alcoholysis step, lipase D from $R$. oryzae immobilized on Accurel MP1000 and Novozym 435 were suitable to produce 2-MAG. However, lipase D quickly lost activity due to pure ethanol. Higher yield was obtained for esterification with lipases D and DF from $R$. oryzae immobilized on Accurel MP1000. Very recently [33], the same team tested the feasibility of producing 2-DHA-MAGs by alcoholysis of cod liver oil with different alcohols catalyzed by Novozym 435 . Esterification of 2-DHA-MAGs with CA was catalyzed by sn-1,3 specific lipase DF. They obtained pure TAGs with mainly the structure CA- 


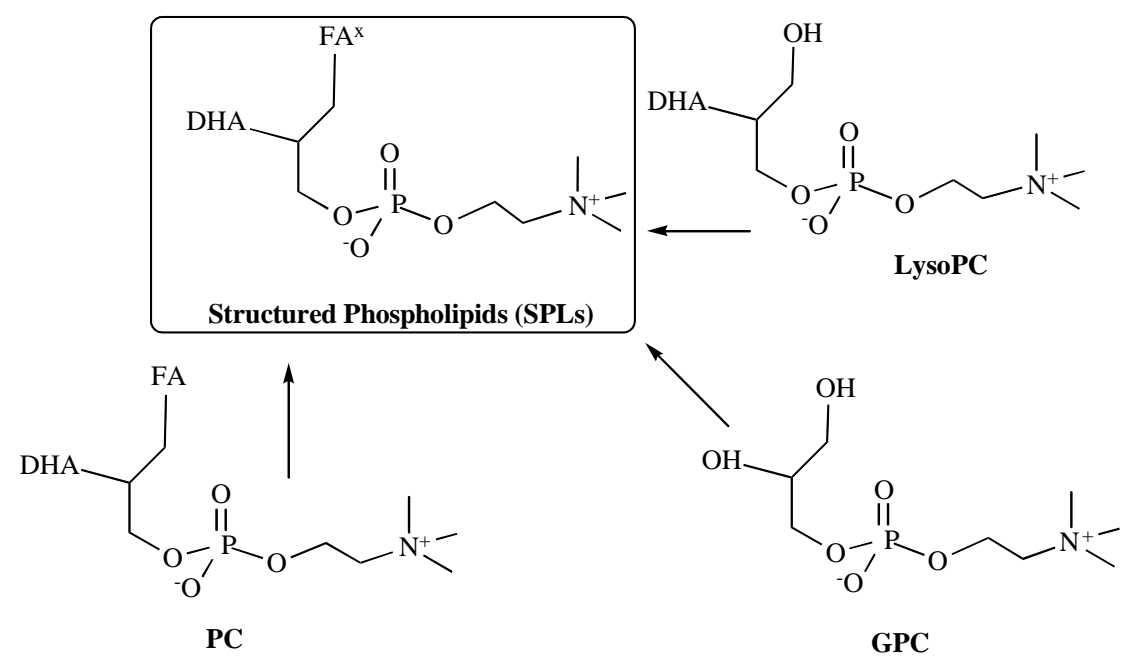

Fig. (3). Scheme for producing structured phospholipids (SPLs). Where GPC $=$ glycerophosphocholine FA ${ }^{x}=$ fatty acid different from FA (in PC) from C2 to longer-chain.

DHA-CA. Zhang et al. [34] tested different reaction routes to produce 1,3-oleoyl-2-DHA-glycerol (ODO). First, they produced 1,3diolein by the reaction of glycerol with oleic acid in the presence of Novozym 435 or Lipozyme RM IM (formerly IM), followed by DHA esterification at the sn-2 position with Novozym 435. A poor yield of TAGs $(9.4 \%)$ was obtained in this second step. Then, they studied the acidolysis of single cell oil rich in DHA (53.7\%) in excess of oleic acid using Novozym 435. The enzyme exhibited a high reaction rate but with a poor regio-specificity with only $39.3 \%$ DHA remaining at the sn-2 position, perhaps due to acyl migration again. So, they used the above-mentioned method via 2-MAG by ethanolysis of single cell oil with Novozym 435, and esterification by oleic acid with Lipozyme RM IM to obtain ODO with good yields, even in large scale.

\subsection{Direct Esterification of Glycerol}

\subsubsection{Chemical Methods}

Traditional synthetic organic chemistry methods used for synthesis of STAGs require a full regio-control and can hardly be undertaken without multi-step protection/de-protection processes. They are based on acylation of 1,3-DAG or 1,2-DAG or via 2MAG. The acylation is generally achieved by reaction of the free acid in presence of 4-dimethylaminopyridine (DMAP) and N,N'dicyclohexylcarbodiimide (DCC). By this method, Endo et al. [35] prepared small amounts of TAGs with three residues of DHA or asymmetrical TAGs with DHA. Instead of DCC, 1-(3dimethylaminopropyl)-3-ethylcarboimide hydrochloride (EDCI) has increasingly been used as an alternative coupling agent.

\subsubsection{Chemo-enzymatic Methods}

Lipases 1,3 acting preferentially, or exclusively, at the external positions of the glycerol backbone, may be used to introduce FAs of selected type at these positions by esterification or transesterification processes. Haraldsson et al. reported successive tests of chemo-enzymatic STAGs synthesis. At first [36], they obtained TAGs with a MCFA $(\mathrm{C} 8, \mathrm{C} 10, \mathrm{C} 12)$ at the sn- 1 and -3 positions and pure EPA or DHA at the internal position with a two-step method. In a first step they obtained 1,3-DAG by enzymatic esterification of glycerol with caprylic, capric and lauric acids in presence of the lipozyme IM. In the subsequent step they introduced
DHA at the sn-2 position of 1,3-DAG by chemical reaction with EDCI and DMAP with a high yield by contrast to Zhang et al. [34]. The nature and the chain length of fatty acyls at the sn- 1 and -3 positions certainly influence the acylation reaction. However, yields of the enzymatic step were moderate due to low regio-selectivity of the enzyme. Later [37], the same authors described the synthesis of TAGs with saturated FAs (C8, C10, C12, C14, C16) at external positions and EPA or DHA at the internal one, and more recently [38], the synthesis of STAGs with short-chain fatty acyls $(\mathrm{C} 2, \mathrm{C} 4, \mathrm{C} 6)$ at the external positions and EPA or DHA at the sn-2 position. They improved the first step by using Novozym 435 instead of Lipozyme RM IM, low temperature $\left(0-4{ }^{\circ} \mathrm{C}\right)$ instead of room temperature, and vinyl esters of FAs instead of their free form. They reported that several studies bring clearly to light that the Rhizomucor miehei lipase is inferior to Candida antartica one to produce 1,3-TAGs. However Zhang et al. [34], reported that Lipozyme RM IM is preferable to acylate glycerol with oleic acid. Also, temperature is a crucial factor in terms of controlling the regio-selectivity of the reaction. Because they detected traces of 1MAG during the lipase reaction, the authors noted that acylmigration and hydrolysis side-reaction could occur due to the presence of low quantities of water in the organic reaction essential for the lipase activity. The acyl-migration process being timedependent, they used vinyl esters which offered a fast and irreversible reaction. In summary, the best conditions for the enzymatic step would favour specific acylation at sn-1 and -3 positions while avoiding acylation at the internal position, as well as acyl migration of sn-1 and -3-acyl substituents and hydrolysis side-reactions.

Fraser et al. [39] reported the synthesis of regio-isomerically enriched symmetrical and asymmetrical TAG isomers containing two palmitoyls and one linoleoyl, linolenoyl, or docosahexaenoyl moieties. By chemical reactions and enzymatic reaction with Candida antartica from glycerol as starting material, they obtained TAGs with good yields. The authors noted a little acyl migration (8-12\%) in the synthesis of the asymmetrical TAGs and a low amount of migration (2-3\%) during synthesis of the symmetrical one. According to the authors, this difference might be due to the 3MAG intermediate in asymmetrical synthesis. Indeed, MAGs are known to isomerize faster than 1,2-DAG or 1,3-DAG that is the intermediate in symmetrical synthesis. 


\subsubsection{Enzymatic Methods}

For some applications, especially when human feeding trials are involved, synthesis of STAGs by non-chemical, purely enzymatic methods is preferred. Along with avoiding toxic solvents and reactants, the mild conditions of enzymatic reactions are especially useful with EPA or DHA which are susceptible to oxidation. Kawashima et al. [24] attempted to synthesize TAGs with CA at the 1,3positions and EPA or DHA at the 2-position by a two-step enzymatic method. In a first step, they synthesized TAGs of PUFA (TriP) by reaction in vacuum of free FAs in presence of Novozym 435 and glycerol. By repeated acidolyses of the resulting TriP with CA using the immobilized Rhizopus lipase, high-purity 1,3dicapryloyl-2-eicosapentaenoyl-glycerol was obtained in good yields whereas 1,3-dicapryloyl-2-docosahexaenoyl-glycerol was synthesized in a poor yield.

Very recently, a novel interesting approach to synthesize symmetric TAGs possessing EPA or DHA at the external positions has been developed. Indeed, Magnusson and Haraldsson [40] suggested that these compounds might be of high value for pharmaceutical and medical purposes since they may be esterified with a drug at the 2-position, and such pro-drugs would acquire the biological properties of both n-3 PUFA and the drug within the same molecule. They might indeed provide improvements to drug's therapeutic value. As we reported above in "chemo-enzymatic methods", Magnusson and Haraldsson [38] have obtained 1,3-DAG consisting in saturated MCFAs at the external positions of the glycerol, with an efficient method using vinyl esters of SFAs as acyl donors in presence of the Novozym 435 lipase. Doing so, EPA or DHA must be activated to be inserted at the external positions of glycerol. In their report, the authors tried to obtain vinyl esters but they noted severe detriments in the framework of the DHA moiety. Other types of activated esters of EPA or DHA were prepared, like 2,2,2trifluoroethylester and oxime ester. The authors considered that oxime esters are the suitable acylating agent since they react faster in a trans-esterification reaction than the corresponding 2-haloyl esters. They obtained with success the desired 1,3-DAGs with EPA or DHA at the external positions in very high yields $(85 \%)$ under conditions similar to those previously described but with the presence of molecular sieves to improve the catalytic process. In the same way, they synthesized 1-O-alkyl,3-acyl-sn-glycerols and their counterparts with EPA or DHA.

By contrast with Magnusson and Haraldsson, $\mathrm{Xu}$ and coworkers [41] optimized the conditions of enzymatic production of the same STAGs containing n-3 PUFAs at the external positions and MCFAs at the sn-2 position by acylating with free n-3 PUFAs or ethyl esters. They established satisfactory quadratic models to find optimal reaction conditions in the production of ABA-type TAGs.

\section{PROPERTY ATTRIBUTES OF MEDIUM AND LONG CHAIN TRIACYLGL YCEROLS}

In dietary oils, the n-3 LC-PUFAs are located at different positions of TAGs, but the stereo-specificity of most native oils favour PUFAs or MUFAs at the sn-2 position. The concept of a SL implies modification of the FA composition and/or FA location on the glycerol backbone, and improvement of the physical and/or physiological properties of dietary lipids.

The lipid structure has been proposed as an important factor to affect the oxidative stability of unsaturated FAs. Endo et al. [35] compared the oxidative stability of randomized and native TAGs from marine oils. They found that fish oils, that contained highly unsaturated TAGs with two or three residues of EPA or DHA, were very unstable. They observed that EPA or DHA located at the 1,2or 2,3-positions of TAGs were more oxidizable than those at the 1,3 -position in response to the 2,2 '-azobis(2-amidinopropane) dihydrochloride oxidation.

The positional distribution of n-3 LC-PUFAs has been shown to influence the oxidative stability as well as the absorption and metabolism of n-3 LC-PUFA oils. DHA has been shown to be more resistant to oxidation and also more efficiently absorbed when located at the sn-2 position of the TAGs. In a recent review, Karupaiah et al. [42] reported that displacing PUFAs or MUFAs from the critical sn-2 position by substitution with SFAs is hypothesized to cause lipid and lipoprotein abnormalities. Studies comparing chain length of SFAs are limited but indicate that saturates are most detrimental at the sn-2 position in animal studies. The authors stated that it is still too early to conclude on the behaviour of unusual positional distribution of FAs in TAGs species originating from oils.

Nagata et al. [43] evaluated the physiological function of STAGs containing MCFAs and PUFAs (EPA-C8-EPA, C8-EPAC8, DHA-C8-C8, and C8-DHA-C8) compared with soybean oil in rats. They observed that serum lipid levels were lowered by SLs, and peri-renal adipose tissue weight was lowered by DHA-C8-C8 and C8-DHA-C8 treatment. Thus, FA species and the differences in intra-molecular distribution of FAs in dietary TAGs affect the nutritional behaviour of dietary lipids.

It has been observed by Yoshida and co-workers [44], that feeding rats with seal oil, in which EPA or DHA is located at the sn-1,3positions of TAGs, more effectively reduced plasma and liver TAG levels through suppression of FA synthesis than fish oil in which EPA and DHA are located at the sn-2 position. Beerman et al. [45] investigated the effects of a dietary test intake containing $72 \%$ MCFAs and $22 \%$ n-3 LC-PUFAs on the fat metabolism in healthy people after 15 days, and found a significant reduction of total plasma TAGs, likely due to increased B-oxidation with synergistic effects of MCFAs and LC-PUFAs. The authors stated that the rapid clearance of blood fat induced by increased stimulation of hepatocytic B-oxidation may represent a new mechanism to loose fat mass. St-Onge et al. [46] reported that consumption of an oil composed of medium-chain TAGs, phytosterols and n-3 FAs, substantially lowered plasma total and LDL cholesterol but did not affect circulating TAGs or HDL cholesterol in overweight women. Recent studies of Shirouchi and al. [47] suggested that feeding porpoise oil containing SLs as a combination of n-3 PUFAs and isovaleric acid (isoC5:0), prevents the development of fatty liver disease through the enhancement of lipoprotein secretion and increase the adiponectin production in obese rats.

\section{SYNTHESIS OF STRUCTURED PHOSPHOLIPIDS (SPLS)}

Although being the main constituents of cell membranes, PLs should not be simply depicted as that. Many advances in lipid biochemistry and molecular biology have led to recognize PLs containing PUFAs such as DHA or EPA, which show important biological activities, as essential molecules and beneficial for human health. Among PUFAs, DHA is especially sensitive to oxidation. A strategy to prevent oxidation is to incorporate DHA into a lipid molecule. Indeed, it has been demonstrated that DHA esterified in PLs is less easily oxidisable than in the forms of either free FA, ethylester, or even TAGs [48-49]. PLs like lysoPLs may be good carriers for 
n-3 PUFAs. Numerous studies showed that supplementation with EPA and DHA in PLs from krill oil, exerts stronger biological effects compared with EPA and DHA in TAGs from fish oil. Rossmeisl et al. [50] recently reported superior metabolic effects of EPA/DHA in PLs from marine fish compared with TAGs in the context of high-fat diet-induced obesity in mice. Vigerust et al. [51] studied krill oil versus fish oil in modulation of inflammation and lipid metabolism in mice transgenic for TNF- and concluded that, when similar doses of n-3 PUFAs are administered, krill oil seems to have a greater potential to promote lipid catabolism, although no differences in the levels of inflammatory markers could be observed. The positive effects of PLs could be partially due to a better bioavailability of EPA or DHA when provided in the form of PLs compared to TAGs or ethyl esters. Lemaitre-Delaunay et al. [52], reported preferential uptake of DHA by human erythrocytes when it was provided as phosphatidylcholine (PC)-DHA compared to TAGDHA and consequently a better potential interest of PC-DHA over TAG-DHA. Study of Wijendran et al. [53] showed that brain arachidonic acid (AA) bioavailability was more than doubled by using PC compared to TAG as carrier of AA. Thies et al. [54] showed a preferential brain incorporation of 2-DHA-LysoPC over unesterified DHA, in the young rat. Bernoud et al. [55] also showed a preference for 2-DHA-LysoPC to cross a re-constituted blood brain barrier compared with unesterified DHA. Also, LysoPC-DHA inhibited the production of pro-inflammatory cytokines in response to LPS whereas unesterified DHA was virtually inactive [56].

There is a growing demand by the food and pharmaceutical industry that has stimulated an extensive search for synthetic methods to produce specific PLs with new combination of FAs. PLs containing EPA or DHA could be directly isolated from fish, but numerous, expansive, and rather laborious extraction and separation procedures are required. On the other hand, numerous publications and patents concern recent progress in physical, chemical, and enzymatic modifications of naturally sourced PLs to enrich certain PL species. Our group has decided to focus on the generation of SPLs, especially DHA-containing PC, using chemical, or biotechnology methods involving enzymes.

In the most common procedure for synthesis of a mixed acid PL, PC containing DHA at the sn-2 position is used as starting material. The FA at the sn-1 position is exchanged with the desired one by trans-esterification using a 1,3-lipase. A combination of a hydrolysis step via a LysoPC intermediate, with subsequent reesterification, is possible as well. A problem with the latter route is the acyl migration within 2-DHA-LysoPC prone to yield the more stable 1-DHA-LysoPC. Another alternative enzymatic procedure uses PC with the desired FA at the sn- 1 position as substrate. PC is thus hydrolyzed by phospholipase $\mathrm{A}_{2}\left(\mathrm{PLA}_{2}\right)$ and re-esterified with DHA by a chemical or enzymatic method using $\mathrm{PLA}_{2}$ favoring the synthetic reaction over hydrolysis. In the two methods, an excess of DHA is required. Another method is possible using glycerophosphocholine (GPC) as starting material, followed by specific esterification at the sn-1 position by the desired FA using a 1,3 lipase, followed by chemical or enzymatic esterification by DHA at the sn-2 position.

\subsection{Enzymatic Incorporation of Fatty Acids at the sn-1 Position}

Haraldsson and coworkers [57] reported results for incorpora- tion of n-3 PUFA by trans-esterification or acidolysis reactions from 1,2-diacyl-PC, using the lipozyme RM IM lipase. They noted that the rate of reactions with zwitterionic head groups is much lower when compared to TAGs. Also, EPA was a better substrate than DHA. The hydrolysis side reactions were much more exten- sive in the inter-esterification reaction than in acidolysis reaction, likely due to a large excess of FA (7x) in the latter reaction. How- ever, excessive amounts of FAs are believed to enhance the acylmigration from the sn-2 to sn-1 positions. The lipase can also influence the acyl migration, particularly when the support is an anionicexchange resin. Poisson et al. [58] showed that after $3 \mathrm{~h}$ of enzymatic hydrolysis of 1-palmitoyl-2-DHA-PC at $40^{\circ} \mathrm{C}$ by a nonimmobilized lipase from Rhizopus oryzae, DHA has totally moved from the sn-2 position. Lowering temperature from $40^{\circ} \mathrm{C}$ to $30^{\circ} \mathrm{C}$ reduced DHA migration by $40 \%$. The authors reported that, by contrast, acyl migration from sn-1 to sn-2 was negligible. Croset et al. [59] studied the isomerization of 2-linoleoyl-LysoPC loaded on albumin and incubated in plasma at physiological $\mathrm{pH}$ and at $37^{\circ} \mathrm{C}$ as a function of time, and reported that after $20 \mathrm{~min}, 75 \%$ isomerization had taken place. This isomerization was prevented at acidic $\mathrm{pH}(<5)$. Rates of isomerization can be different in acidolysis or esterification process using immobilized lipases in organic solvent or solvent-free.

Other examples of FA exchange between the sn- 1 and sn-2 positions of PLs by one step (acidolysis) or two-step processes have been reviewed by Adlercreutz et al. [60]. The one-step reaction or two-step approach via LysoPC intermediate obtained by hydrolysis or alcoholysis, and re-esterification with the same enzyme give similar yields; however time is longer for the acidolysis reaction. The authors also reported that incorporation of FAs into the sn-1 position was more favorable than to the sn-2 position because the lipase can be used at much lower water activity than $\mathrm{PLA}_{2}$, which is in favor of the esterification reaction. Acylation by EPA and DHA mixture using pancreatic $\mathrm{PLA}_{2}$ realized in organic solvents needed a water activity of 0.2 and high FA concentration. Some acylation by EPA and DHA indicated that glycerol could be a good solvent, and better yields were obtained with addition of some amino acids in the reaction mixture [61]. When pure EPA was added in presence of glycine + alanine, the EPA-PL amounted 49\%, compared with 19\% DHA-PL when 54\% DHA was used.

Conversion of 2-palmitoyl-LysoPC in PC by regio-selective incorporation of EPA and DHA from fish oil to the sn-1 position was investigated using five different immobilized lipases and PLA ${ }_{1}$ [62]. Results showed that lipases discriminated against EPA and especially DHA. Indeed, Candida antartica was the only lipase that incorporated significant amounts of DHA. For incorporation of DHA to the sn-2 position, PLA $_{2}$ was used but the low reaction and yield showed that this reaction was not efficient. Chemical coupling of 1-palmitoyl-LysoPC and DHA was preferred. The authors reported low yield $(<30 \% \mathrm{~mol})$ of LysoPC conversion with immobilized $\mathrm{PLA}_{1}$. However, some recent papers reported production of structured PC with immobilized PLA 1 [63-64]. First, one study reported $\mathrm{PLA}_{1}$-catalyzed acidolysis of $\mathrm{PC}$ by mixture of FAs from fish oil containing mainly DHA, EPA and DPA, in solvent-free conditions. From their results obtained by modifying water activity, vacuum and enzyme loading, the authors concluded that the synthesis protocols leading to large proportions of n-3 PUFA residues in PC and LysoPC lead simultaneously to low yields of these compounds. The second study pointed out that the high substrate purity used affected the equilibrium of the acidolysis reaction and clearly favored the acylation by the immobilized PLA 1 . Indeed, selection of optimum synthesis conditions may well depend on the specific applications of interest. Vikberg et al. [65] studied the acyl modifi- 
cation of the sn-2 position of PC from soybean by acidolysis using immobilized $\mathrm{PLA}_{2}$ and different FAs as acyl donors. Under the same conditions, different FAs result in different incorporation into PLs or different yields. They described the highest degree of incorporation for conjugated linoleic acid (39\%) followed by CA $(25 \%)$ and DHA (20\%). When using DHA as acyl donor, the yield $(21 \%)$ was also lower than for CA (29\%).

A chemo-enzymatic synthesis of a particular structured PC containing an acetyl group at the sn-1 position to mimic 2-DHALysoPC, and prevent the acyl migration has been reported [66]. 2DHA-LysoPC was prepared from 1-stearoyl-2-DHA by enzymatic hydrolysis using the Rhizopus arrhizus lipase, and was acetylated by acetic anhydride into 1-acetyl-2-DHA-PC. This new structured PC, named AceDoPC, has been produced with more important quantities and better yield by a one step-trans-esterification of microalgae DHA-containing PC using lipozyme from Mucor miehei in a bioreactor [67].

\section{INTEREST OF MODIFIED PHOSPHOLIPIDS}

To date, few SPLs have found wide industrial applications. This may be due to the lack of studies to evaluate the properties of novel compounds. SPLs from acidolysis of soybean lecithin with oil enriched with omega-3 FAs (containing 51.4\% EPA/DHA), have been recently shown to reduce atherosclerosis risk in rats fed an atherogenic diet [68]. New structured molecules seem to be promising, like AceDoPC which showed a therapeutic potential for acute stroke treatment. Indeed, the authors that considered this new molecule as a brain-targeting form of DHA assessed in vivo neuroprotection conferred by DHA compared with AceDoPC in experimental stroke in rats, by using multimodal MRI to monitor the brain damage. They showed a more important neuro-protective effect of AceDoPC than unesterified DHA, that could be partly due to decrease of lipid peroxidation [69].

Novel PLs could combine the FA'-s and the drug'-activities in the same molecule for simultaneous delivery of the two agents. Zerouga et al. [70] reported a potential use of PC with DHA at the sn-1 position and the cytotoxic drug methotrexate at the sn-2 position, in cancer chemotherapy.

Whereas the anti-inflammatory actions of hydroxylated derivatives of DHA, such as neuroprotectin D1, have been reported, recent literature showed that 1-DHA-2-LysoPC and 17-hydroperoxyDHA-2-LysoPC [71] exhibit anti-inflammatory effects as well. It seems that oxygenated metabolites derived from DHA, like resolvins and protectins may play a significant role as they have potent anti-inflammatory and immuno-regulatory actions [72-73]. Synthesis of novel PLs containing these compounds could have a great interest.

\section{CONCLUSION}

For a long time, intense research concluded that n-3 PUFAs possess nutritional essentiality, and beneficial effects in cardiovascular disease, hypertension, and inflammation. There has been much interest in recent years for a promising role of DHA in cognition, and prevention of neurodegenerative disorders. However, many results are conflicting, likely due to the quantity of administered EPA or DHA, the purity of FAs and to the form used for their administration. Structured lipids could perhaps help to choose between PL, TAG or unesterified forms.

\section{CONFLICT OF INTEREST}

The author(s) confirm that this article content has no conflict of interest.

\section{ACKNOWLEDGEMENTS}

The authors thank the support of Inserm, ANR, the French Ministry of Education and Research Education through INSA-Lyon, and a grant from the LISA Carnot institute.

\section{REFERENCES}

[1] Nakamura, T.; Tokunaga, K.; Shimomura, L.; Nishida, M.; Yoshida, S.; Kotani, K.; Islam, A.H.; Keno, Y.; Kobatake, T.; Nagai, Y.; Fujioka, S.; Ta- rui, S.; Matsuzawa, Y. Contribution of visceral fat accumulation to the de- velopment of coronary artery disease in non-obese men. Atherosclerosis,

1994, 107, 239-246.

[2] Formiguera, X.; Canton, A. Obesity: Epidemiology and clinical aspects. Best. Pract. Res. Clin. Gastroenterol., 2004, 18, 1125-1146.

[3] Solfrizzi, V.; Panza, F.; Frisardi, V.; Seripa, D.; Logroscino, G.; Imbimbo, B.P.; Pilotto, A. Diet and Alzheimer's disease risk factors or prevention: the current evidence. Expert. Rev. Neurother., 2011, 11, 677-708.

[4] Vessby, B. Dietary fat, fatty acid composition in plasma and the metabolic syndrome. Curr. Opin. Lipidol., 2003, 14, 15-19.

[5] Chen, S.; Chen, R. Docosahexaenoic acid-containing phospholipids and triglycerides based nutritional supplements. Recent Pat. Food. Nutr. Agric.,

2010, 2, 213-220.

[6] Giudetti, A.M.; Cagnazzo, R. Beneficial effects of n-3 PUFA on chronic

airway inflammatory diseases. Prostaglandins Other. Lipid. Mediat., 2012,

99, 57-67.

[7] Jump, D.B. N-3 polyunsaturated fatty acid regulation of hepatic gene transcription. Curr. Opin. Lipidol., 2008, 19, 242-247.

[8] Souza, L.L.; Cordeiro, A.; Oliveira, L.S.; de Paula, G.S.; Faustino,

L.C.;

Ortiga-Carvalho, T.M.; Oliveira, K.J.; Pazos-Moura, C.C. Thyroid hormone contributes to hypolipidemic effect of polyunsaturated fatty acids from fish oil: in vivo evidence for cross talking mechanisms. J. Endocrinol., 2011, 211, 65-72.

[9] De Caterina, R.; Madonna, R .; Bertolotto, A .; Schmidt, E.B. N-3 fatty acids in the treatment of diabetic patients. Diabetes Car., 2007, 30, 1012-1026.

[10] Coste, T.; Gerbi, A.; Vague, P.; Pieroni, G.; Raccah, D. Neuroprotective effect of docosahexaenoic acid-enriched phospholipids in experimental dia- betic neuropathy. Diabetes, 2003, 52, 2578-2585.

[11] Mozaffarian, D.; Wu, J. Omega-3 fatty acids and cardiovascular

disease.

[12] Corsetto, P.A.; Montorfano, G.; Zava, S.; Jovenitti, I.E.; Cremona, A.; Berra, B.; Rizzo, A.M. Effects of n-3 PUFA on breast cancer cells through their incorporation in plasma membranes. Lipids Health Dis., 2011, 12,10-73.

[13] Guo, Z.; Vikbjerg, A.F.; Xu, X.B. Enzymatic modification of phospholipids for functional applications and human nutrition. Biotechnol. Adv., 2005, 23, 203-259.

[14] Doig, S.D.; Diks, R.M.M. Toolbox for exchanging constituent fatty acids in lecithin. Eur. J. Lipid Sci. Technol., 2003, 105, 359-367.

[15] Timmermann, F. Functional food and lipids. Oils Fats. Int., 2002, 18, 18-19.

[16] Babayan, V.K. Medium chain triglycerides and structured lipids,

Lipids,

Trivedi, R.; Singh, R. P. Modification of oils and fats to produce structured lipids. Journal of oleo Science, 2005, 54, 423-430.

[18] Marangoni, A.G.; Rousseau, D. Chemical interesterification. Food Lipids, 1998, 251-281.

[19] Haraldsson, G.G. The application of lipases for preparing various lipids enriched with omega-3 fatty acids. Rit. Fiskideildar., 1999, 16, 97-105.

[20] Haraldsson, G.; Almarsson, O. Studies on the positional specificity of lipase from Mucor miehei during interesterification reactions of cod liver oil with n-3 polyunsaturated fatty acid and ethyl ester concentrates. Acta Chem. Scand., 1991, 45,723-730.

[21] Huang, K.H.; Akoh, C. Optimization and scale-up of enzymatic synthesis of structured lipids using RSM. J. Food Sci., 1996, 61, 137-141.

[22] Akoh, C.C.; Moussata, C.O. Characterization and oxidative stability of enzymatically produced fish and canola-oil-based structured lipids. $J$. Am. Oil Chem. Soc., 2001, 78, 25-30.

[23] Jennings, B.; Akoh, C.C. Enzymatic modification of triacylglycerols of high eicosapentaenoic and docosahexaenoic acids content to produce structured lipids. J. Am. Oil. Chem. Soc., 1999, 76, 1133-1137.

[24] Kawashima, A.; Shimada, Y.; Yamamoto, M.; Sugihara, A.; Nagao, T.; Komemushi, S.; Tominaga, Y. Enzymatic synthesis of high-purity structured lipids with caprylic acid at 1,3-positions and polyunsaturated fatty acid at 2position. J. Am. Oil Chem. Soc., 2001, 78, 611-616.

[25] Iwasaki, Y.; Han, J.J.; Narita, M.; Rosu, R.; Yamane, T. Enzymatic synthesis of structured lipids rom single cell oil of high docosahexaenoic acid content. J. Am. Oil Chem. Soc., 1999, 76, 563-669. 
[26] Shimada, Y.; Sugihara, A.; Maruyama, T.; Nagao, T.; Nakayama, H.; Na- kano, H.; Tominaga, Y. Production of structured lipid containing docosahex- aenoic and caprylic acids using immobilized Rhizopus delemar lipase. J. Ferment. Bioeng., 1996, 81, 299-303.

[27] Hamam, F.; Shahidi, F. Synthesis of structured lipids via acidolysis of docosahexaenoic acid single cell oil (DHASCO) with capric acid. J. Agric. Food Chem., 2006 54, 2900-2906.

[28] Xu, X.; Skands,A.; Jonsson, G. Production of structured lipids by lipase- catalysed interesterification in an ultrafiltration membrane reactor. Biotech-nology. Letters, 2000, 22, 1667-1671.

[29] Garcia, H.; Arcos, J.; Ward, D.; Hill, C. Synthesis of glycerides containing n3 fatty acids and conjugated linoleic acid by solvent-free acidolysis of fish

oil. Biotechnol. Bioeng., 2000, 587-591.

[30] Irimescu, R.; Furihata, K.; Hata, K.; Iwasaki, Y.; and Yamane, T. Utilization of reaction medium-dependent regiospecificity of Candida antarctica lipase (Novozym 435) for the synthesis of 1,3-dicapryloyl-2-docosahexaenoyl. (or eicosapentaenoyl) glycerol. J. Am. Oil Chem. Soc., 2001, 78, 285-290.

[31] Irimescu, R.; Furihata, K.; Hata, K.; Iwasaki, Y.; and Yamane, T. Two-step enzymatic synthesis of docosahexaenoic acid-rich symmetrically structured triacylglycerols via 2-monoacylglycerols. J. Am Oil Chem. Soc., 2001, 78

743-748.

[32] Munio, M.; Robles, A.; Esteban, L.; Gonzalez, P.; Molina, E. Synthesis of structured lipids by two enzymatic steps: Ethanolysis of fish oils and esterifi- cation of 2-monoacylglycerols. Process Biochemistry, 2009, 44, 723730.

[33] Rodríguez, A.; Esteban, L.; Martín, L.; Jiménez, M.J.; Hita, E.; Castillo, B.; González, P.A.; Robles, A. Synthesis of 2-monoacylglycerols and structured triacylglycerols rich in polyunsaturated fatty acids by enzyme catalyzed reactions. Enzyme Microb. Technol., 2012, 51, 148-155.

[34] Zhang, H.; Önal, G.; Wijesundera, C.; Xu X. Practical synthesis of 1,3-oleoyl 2-docosahexaenoyl-glycerol by lipase-catalyzed reactions: An evaluation of different reaction routes Process. Biochemistry, 2009, 44, 534-539.

[35] Endo, Y.; Hoshizaki, S.; Fujimoto, K. Oxidation of synthetic triacylglycerols containing eicosa-pentaenoic and docosahexaenoic acids: Effect of oxidation system and triacylglycerol structure. J. Am. Oil.Chem. Soc., 1997, 74, 10411045 .

[36] Haldorsson, A.; Magnusson, C.; Haraldsson, G.G. Chemoenzymatic synthesis of structured triacylglycerols. Tetrahedron. Lett., 2001, 42, 7675-7677.

Haldorsson, A.; Magnusson, C.; Haraldsson, G.G. Chemoenzymatic synthesis of structured triacylglycerols. Tetrahedron, 2003, 59, 9101-9109.

[38] Magnusson, C.; Haraldsson, G.G. Chemoenzymatic synthesis of structured triacylglycerols possessing short-chain fatty acids. Tetrahedron, 2010, 66 , 2728-2731.

[39] Fraser, B.; Perlmutter, P.; Wijesundera, C. Practical syntheses of triacylglycerol regioisomers containing long-chain polyunsaturated fatty acids. J. Amer. Oil. Chem. Soc., 2007, 84,11-21.

[40] Magnusson, C.D.; Haraldsson, G.G. Activation of n-3 polyunsaturated fatty acids as oxime esters: a novel approach for their exclusive incorporation into the primary alcoholic positions of the glycerol moiety by lipase. Chem. Phys. Lipids, 2012, 165, 712-720.

[41] Chen, B.; Zhang, H.; Cheong, L.Z.; Tan, T.; Xu, X. Enzymatic production of ABA-type structured lipids containing omega 3 and medium-chain fatty acids: Effects of different acyl donors on the acyl migration rate. Food. Bioprocess. Technol., 2012, 5, 541-547.

[42] Karupaiah, T.; Sundram, K. Effects of stereospecific positioning of fatty acids in triacylglycerol structures in native and randomized fats: a review of their nutritional implications. Nutr. Metab., 2007, 4, 16.

[43] Nagata, J.; Kasai, M.; Negishi, Saito, M. Effects of structured lipids

containing eicosapentaenoic or docosahexaenoic acid and caprylic acid on serum and liver lipid profiles in rats. BioFactors, 2004, 22,157-160.

[44] Yoshida, H.; Kumamaru, J.; Mawatari, M.; Ikeda, I.; Imaizumi, K.; Tsuji, H.; Seto, A. Lymphatic absorption of seal and fish oils and their effect on lipid metabolism and eicosanoid production in rats. Biosci. Biotech. Biochem.,

1996, 60, 1293-1298

[45] Beermann, C.; Jelinek, J ; Reinecker, T.; Hauenschild, A.; Boehm, G.; Klör, H-U. Short term effects of dietary medium-chain fatty acids and n-3 long- chain polyunsaturated fatty acids on the fat metabolism of healthy volun- teers. Lipids Health Dis., 2003, 2, 1-10.

[46] St-Onge, M.P.; Lamarche, B.; Mauger, F; Jones, P.J. Consumption of

functional oil rich in phytosterols and medium-chain triglyceride oil im- proves plasma lipid profile in men. J. Nutr., 2003, 133,1815-1820.

[47] Shirouchi, B.; Nagao, K.; Furuya, K.; Nagai, T.; Ichioka, K.; Tokairin, S.; Iida, Y.; Yanagita,T. Physiological functions of iso-type short-chain fatty acid and omega 3 polyunsaturated fatty acids containing oil in obese OLETF rats. J. Oleo. Sci, 2010, 6, 299-305.

[48] Song, J.H.; Inoue,Y.; Miyazawa, T. Oxidative stability of docosahexaenoic acid-containing oils in the form of phospholipids, triacylglycerols and ethyl esters. Biosci. Biotech. Biochem., 1997, 61, 2085-2088.

[49] Lyberg, A.M.; Fasoli, E.; Adlercreutz, P. Monitoring the oxidation of docosahexaenoic acid in lipids. Lipids, 2005, 969-979.

[50] Rossmeisl, M.; Jilkova, Z .; Kuda, O.; Jelenik, T.; Medrikova, D.; Stankova, B.; Kristinsson, B.; Haraldsson, G.G.; Svensen, H.; Stoknes, I .; Sjövall, P.; Magnusson, Y.; Balvers, M.; Verhoeckx, K.; Tvrzicka, E.; Bryhn, M.; Kopecky, J. Metabolic effects of n-3 PUFA as phospholipids are superior to triglycerides in mice fed a high-fat diet: possible role of endocannabinoids. PLoS. One, 2012, 7, 1-13.

[51] Vigerust, N.; Bjorndal, B.; Bohov, P.; Brattelid, T.; Svardal, A.; Berge, R Krill oil versus fish oil in modulation of inflammation and lipid metabolism in mice transgenic for TNF- . Eur. J. Nutr., 2012, Aug 25.

[52] Lemaitre-Delaunay, D.; Pachiaudi, C.; Laville, M. Pousin, J.; Armstrong, M.; Lagarde, M. Blood compartmental metabolism of docosahexaenoic acid (DHA) in humans after ingestion of a single dose of $\left[{ }^{13} \mathrm{C}\right] \mathrm{DHA}$ in phosphatidylcholine. J. Lipid. Res., 1999, 40, 18671874

[53] Wijendran, V.; Huang, M.C.; Diau, G.Y.; Boehm, G.; Nathanielsz,

Brenna, J.T. Efficacy of dietary arachidonic acid provided as triglyceride or phospholipid as substrates for brain arachidonic acid accretion in baboon neonates. Pediatr. Res., 2002, 51, 265-272.

[54] Thies, F.; Pillon, P.; Moliere, P.; Lagarde, M.; Lecerf, J. Preferential incorporation of sn-2 lysoPC DHA over unesterified DHA in the young rat brain

Am. J. Physiol., 1994, R1273-R1279.

[55] Bernoud, N.; Fenart, L.; Moliere, P.; Dehouck, M.P.; Lagarde, M.; Cecchelli, M.; Lecerf, J. Preferential transfer of 2-docosahexaenoyl-1lysophosphatidylcholine through an in vitro blood-brain barrier over unesterified docosahexaenoic acid. J. Neurochem., 1999, 72, 338-345.

[56] Jim, M.; Hung, N.; Yoo, J.; Kim, M.; Sok, D-E. Suppressive effect of docosahexaenoyl-lysophosphatidylcholine and hydroxydocosahexaenoyl- lysophosphatidylcholine on levels of cytokines in spleen of mice treated with lipopolysaccharide. Eur. J .Lipid Sci. Technol., 2012, 114, 114-122.

[57] Haraldsson, G.G.; Thorarensen, A. Preparation of phospholipids highly enriched with n-3 polyunsaturated fatty acids by lipase. J. Am. Oil Chem. Soc., 1999, 76, 1143-1149.

[58] Poisson, L.; Devos, M.; Godet, S.; Ergan, F.; Pencreac'h, G. Acyl migration during deacylation of phospholipids rich in docosahexaenoic acid (DHA): an enzymatic approach for evidence and study. Biotechnol. Lett., 2009, 31, 743749.

[59] Croset, M.; Brossard, N.; Polette, A.; Lagarde, M. Caracterization of plasma unsaturated lysophosphatidylcholine in human and rat. Biochem. J., 2000

$345,61-67$.

[60] Adlercreutz, P.; Lyberg, A.M.; Adlercreutz, D. Enzymatic fatty acid exchange in glycero- phospholipids. Eur. J. Lipid Sci. Technol., 2003, 105 ,

638-645

[61] Tanaka, T.; Isezaki, T.; Nakano, H.; Iwasaki, Y. Synthesis of phospholipids containing polyunsaturated fatty acids by phospholipase $\mathrm{A}_{2}$-mediated esterification with food-compatible reagents. J. Oleo. Sci.; 2010, 59, 375 -

380

[62] Lyberg, A.M.; Adlercreutz, D.;Adlercreutz, P. Enzymatic and chemical synthesis of phosphatidylcholine regioisomers containing eicosapentaenoic acid or docosahexaenoic acid. Eur J Lipid Sci. Technol., 2005, 107, 279-280.

[63] Kim, I.H.; Garcia, H.; Graham Hill, C. Phospholipase $A_{1}$-catalyzed synthesis of phospholipids enriched in $\mathrm{n}-3$ polyunsaturated fatty acid residues Enz. Microb. Technol., 2007,40,1130-1135.

[64] Baeza Jiménez, J.; Noriega-Rodríguez, H.; García, H.; Otero, C. Structured phosphatidylcholine with elevated content of conjugated linoleic acid: Optimization by response surface methodology. Eur. J. Lipid Sci. Technol., 2012,114, 1261-1267.

[65] Vikbjerg, A.F.; Mu, H.; Xu, X. Synthesis of structured phospholipids by immobilized phos-pholipase $\mathrm{A}_{2}$-catalyzed acidolysis. J. Biotechnol., 2007

$128,545-554$

[66] Polette, A.; Deshayes, C.; Chantegrel, B.; Croset, M.; Armstrong, JM.; Lagarde, M. Synthesis of acetyl,docosahexaenoyl-glycerophosphocholine and its characterization using nuclear magnetic resonance. Lipids, 1999, 34, 1333-37.

[67] Lagarde, M.; Guichardant, M.; Picq, M.; Michaud, S.; Doutheau, A. Method for preparing acetyl,docosahexaenoyl-glycerophosphocholine and use thereof for the delivery of polyunsaturated fatty acids. WIPO Patent Application WO/068413/2008.

[68] Maligan, J.M.; Estiasih, T.; Kusnadi, J. Structured phospholipids from commercial soybean lecithin containing omega-3 fatty acids reduces atherosclerosis risk in male Sprague dawley rats which fed with an atherogenic diet. World academy of science, Engineering and technology,

2012, 69, 552-558

[69] Chauveau, F.; Cho, T.H.; Perez, M.; Guichardant, M.; Riou, A.; Aguettaz, P.; Picq, M.; Lagarde, M.; Berthezene, Y.; Nighoghossian, N.; Wiart, M. Braintargeting form of docosahexaenoic acid for experimental stroke treatment: MRI evaluation and anti-oxidant impact. Curr. Neurovasc. Res., 2011, 8, 95102 .

[70] Zerouga, M.; Stillwell, M.; Jenski, L. Synthesis of a novel phosphatidylcholine conjugated to docosahexaenoic acid and methotrexate that inhibits cell proliferation. Anti-Cancer Drugs, 2002, 3, 301-311.

[71] Huang, L.; Hung, N.; Sok, D-E.; Kim, M. Lysophosphatidylcholine containing docosahexaenoic acid at the sn-1 position is anti-inflammatory. Lipids, 2010, 45, 225-236. 
[72] Serhan, C.N.; Petasis, NA. Resolvins and protectins in inflammation resolution. Chem. Rev., 2011 111, 5922-5943.

[73] Kohli, P.; Levy, B.D. Resolvins and protectins mediating solutions to inflammation. Brit. J. Pharm., 2009, 158, 960-

971. 